\title{
Use of Non-Vitamin K Antagonist Oral Anticoagulants Among Patients with Nonvalvular Atrial Fibrillation and Multimorbidity
}

\author{
Steven Deitelzweig · Allison Keshishian · Amiee Kang • \\ Amol D. Dhamane $\cdot$ Xuemei Luo $\cdot$ Christian Klem $\cdot$ Lisa Rosenblatt \\ Jack Mardekian · Jenny Jiang $\cdot$ Huseyin Yuce $\cdot$ Gregory Y. H. Lip
}

Received: January 28, 2021 / Accepted: March 24, 2021 / Published online: May 7, 2021

(C) The Author(s) 2021

\section{ABSTRACT \\ Introduction: Non-valvular atrial fibrillation (NVAF) is often accompanied by multiple comorbid conditions, which increase the asso- ciated risks and complexity of patient man- agement. This study evaluated the risk of stroke/systemic embolism (SE) and major bleeding (MB) among multimorbid patients with NVAF who were prescribed non-vitamin $\mathrm{K}$}

Supplementary Information The online version contains supplementary material available at https:// doi.org/10.1007/s12325-021-01724-8.

S. Deitelzweig $(\bowtie)$

Department of Hospital Medicine, Ochsner Health,

Ochsner Clinic Foundation, 1514 Jefferson

Highway, 11th floor, Hospital Medicine, New

Orleans, LA 70121, USA

e-mail: sdeitelzweig@ochsner.org

S. Deitelzweig

Ochsner Clinical School, The University of

Queensland School of Medicine, New Orleans, LA, USA

A. Keshishian

STATinMED Research, Ann Arbor, MI, USA

A. Keshishian · H. Yuce

New York City College of Technology, City

University of New York, New York, NY, USA

A. Kang · A. D. Dhamane - C. Klem - L. Rosenblatt .

J. Jiang

Bristol-Myers Squibb Company, Lawrenceville, NJ,

USA antagonist oral anticoagulants (NOACs) or warfarin.

Methods: A retrospective study of patients with NVAF and high multimorbidity who initiated apixaban, dabigatran, rivaroxaban, or warfarin from 1 January 2013 to 30 September 2015 was conducted using five insurance claims databases. Multimorbidity was defined as six or more comorbid conditions, and 1:1 propensity score matching (PSM) was conducted between the NOAC-warfarin and NOAC-NOAC cohorts. Cox proportional hazard models were used to evaluate the hazard ratios of stroke/SE and MB.

X. Luo

Pfizer, Inc., Groton, CT, USA

J. Mardekian

Pfizer, Inc., New York, NY, USA

G. Y. H. Lip

Liverpool Centre for Cardiovascular Science,

University of Liverpool and Liverpool Heart \& Chest Hospital, Liverpool, UK

G. Y. H. Lip

Aalborg Thrombosis Research Unit, Department of Clinical Medicine, Aalborg University, Aalborg, Denmark 
Results: Of the NVAF population $(n=466,991)$, $33.4 \% \quad(n=155,959)$ had multimorbidity, including 36,921 apixaban, 10,248 dabigatran, 45,509 rivaroxaban, and 63,281 warfarin patients. Compared to warfarin, apixaban and rivaroxaban were associated with a lower risk of stroke/SE (hazard ratio [HR] 0.63, 95\% CI 0.54-0.74; HR 0.70, 95\% CI 0.64-0.77, respectively). Apixaban and dabigatran were associated with a lower risk of MB (HR 0.61, 95\% CI 0.56-0.67; HR 0.75, 95\% CI 0.66-0.86, respectively) and rivaroxaban was associated with a higher risk of MB (HR 1.06, 95\% CI 1.01-1.12) compared to warfarin.

Conclusions: Among patients with NVAF and six or more comorbid conditions, NOACs were associated with varying risk of stroke/SE and $\mathrm{MB}$ compared to warfarin and to each other. Rather than a "one drug fits all" approach, our results may be useful for appropriate OAC treatment for multimorbid patients.

Keywords: Anticoagulation; Bleeding; Multimorbidity; Outcomes; Stroke

\section{Key Summary Points}

Why carry out this study?

The presence of multiple comorbid conditions increases the risk of adverse clinical outcomes in patients with AF.

This study evaluates the safety and effectiveness of non-vitamin $\mathrm{K}$ antagonist oral anticoagulants (NOACs) versus warfarin among patients with $\mathrm{AF}$ and multiple comorbid conditions.

\section{What was learned from the study?}

In this multimorbid subgroup of patients with $\mathrm{AF}$, NOACs were associated with varying risks of stroke/SE and major bleeding compared to warfarin.

These results may help inform treatment selection in this high-risk subgroup of patients with AF by allowing clinicians to choose the safest and most effective treatment according to the patient comorbidity burden.

\section{DIGITAL FEATURES}

This article is published with digital features, including a summary slide, to facilitate understanding of the article. To view digital features for this article go to https://doi.org/10.6084/ m9.figshare.14261612.

\section{INTRODUCTION}

Atrial fibrillation (AF) is the most common serious cardiac arrhythmia, affecting $2-3 \%$ of the population in North America [1]. Previously, vitamin $\mathrm{K}$ antagonists, such as warfarin, were the most commonly prescribed therapeutic agents for AF. However, the needs for periodic patient monitoring and frequent dose adjustment increase the patient burden of treatment [2]. Non-vitamin $\mathrm{K}$ antagonist oral anticoagulants (NOACs: apixaban, dabigatran, edoxaban, and rivaroxaban) offer a non-inferior, safe, effective treatment choice for stroke prevention in patients with non-valvular atrial fibrillation (NVAF) without the restrictions of conventional therapy $[3,4]$.

Multimorbidity, often defined as the coexistence of two or more comorbid conditions, may provide a proxy for identifying chronic disease burden and functional status and may influence treatment selection [5]. More than two-thirds of Medicare patients have two or more comorbidities; the median number is six, with $\mathrm{AF}$ being the 11th most common [6]. Multimorbidity is associated with an increased risk of AF, cerebrovascular events, and mortality, with the risk increasing in accordance with the number of long-term conditions [7, 8]. Furthermore, patients with multimorbidity receiving oral anticoagulation therapy are associated with an increased risk of stroke relative to patients without multimorbidity who receive anticoagulation therapy [6]. Indeed, ad hoc results from the ARISTOTLE trial found that the risk of major bleeding (MB) rises by approximately $15 \%$ for each singular comorbidity [9]. However, despite the need for a safe treatment in this patient population, anticoagulants are generally underused-fewer than half of older adults with $\mathrm{AF}$, who are generally multimorbid, receive 
anticoagulants even without contraindications [6].

There have been very few observational studies comparing NOACs and warfarin among patients with NVAF and multimorbidity. This ARISTOPHANES (Anticoagulants for Reduction In Stroke: Observational Pooled Analysis on Health Outcomes and Experience of Patients; NCT03087487) sub-study compared the risk of stroke/SE and MB among multimorbid patients with NVAF who were newly prescribed apixaban, dabigatran, rivaroxaban, or warfarin.

\section{METHODS}

\section{Data Sources}

This retrospective observational analysis utilized Medicare fee-for-service data from the US Centers for Medicare \& Medicaid Services (CMS) and data from four US commercial claims databases: the IBM MarketScan ${ }^{\circledR}$ Commercial Claims and Encounter Database, the IQVIA PharMetrics Plus ${ }^{\mathrm{TM}}$ Database, the Optum Clinformatics $^{\mathrm{TM}}$ Data Mart, and the Humana Research Database. Previously published articles include detailed descriptions of the datasets, the rationale for the pooling process, and the approaches to minimizing potential patient record duplicates across data sources $[10,11]$. To avoid potential duplicates, patients with Medicare supplemental plans in MarketScan and PharMetrics data were excluded from the study because those patients may also have Medicare Part A and Part B coverage. For Optum and Humana, beneficiaries aged 65 years or more are enrolled in Medicare Advantage plans but are not covered in Medicare data. There is a possibility that employer-based commercial claims datasets may contain duplicate patient records when pooled together; however, the number of duplicates is likely to be small at an estimated $0.5 \%$.

\section{Patient Selection}

For this study, multimorbidity was defined as the presence of six or more comorbid conditions, not including $\mathrm{AF}$, among the 17 conditions listed in Tables 1 and 2 [9], based on both the distribution of comorbidities as well as the ARISTOTLE multimorbidity subgroup analysis, the only prior published analysis based on a phase 3 randomized controlled trial (RCT) for NOACs in a multimorbid population [9]. The present study utilized the same count for high multimorbidity as seen in the ARISTOTLE subgroup analysis. Patients with NVAF and multimorbidity were selected if they had at least one pharmacy claim for apixaban, dabigatran, rivaroxaban, or warfarin between 1 January 2013 to 30 September 2015 (identification period). Edoxaban was not included in the final analysis because of the small sample size. For patients with a NOAC claim during the identification period, the first NOAC prescription date was designated as the index date; for patients with no NOAC claim during the identification period, the first warfarin prescription date was designated as the index date. Patients were required to have an AF diagnosis before the index date and have continuous medical and pharmacy health plan enrollment for at least 12 months pre-index date.

Patients treated with an OAC within 12 months pre-index date were excluded. Patients were also excluded if they had evidence of valvular heart disease, venous thromboembolism, transient $\mathrm{AF}$, heart valve replacement/transplant during the baseline period, pregnancy during the study period, or hip or knee replacement surgery within 6 weeks preindex date. Additional exclusion criteria are shown in Fig. 1.

\section{Outcome Measures}

The primary outcomes measured were time to first stroke/SE and time to first MB. Stroke/SE was stratified by ischemic stroke, hemorrhagic stroke, and systemic embolism (SE). MB was stratified by gastrointestinal (GI) bleeding, intracranial hemorrhage, and bleeding at other key sites (Supplementary Table S1) [12, 13]. Outcomes were based on hospitalizations with stroke/SE or MB as the principal or first-listed diagnosis. The follow-up period ranged from 


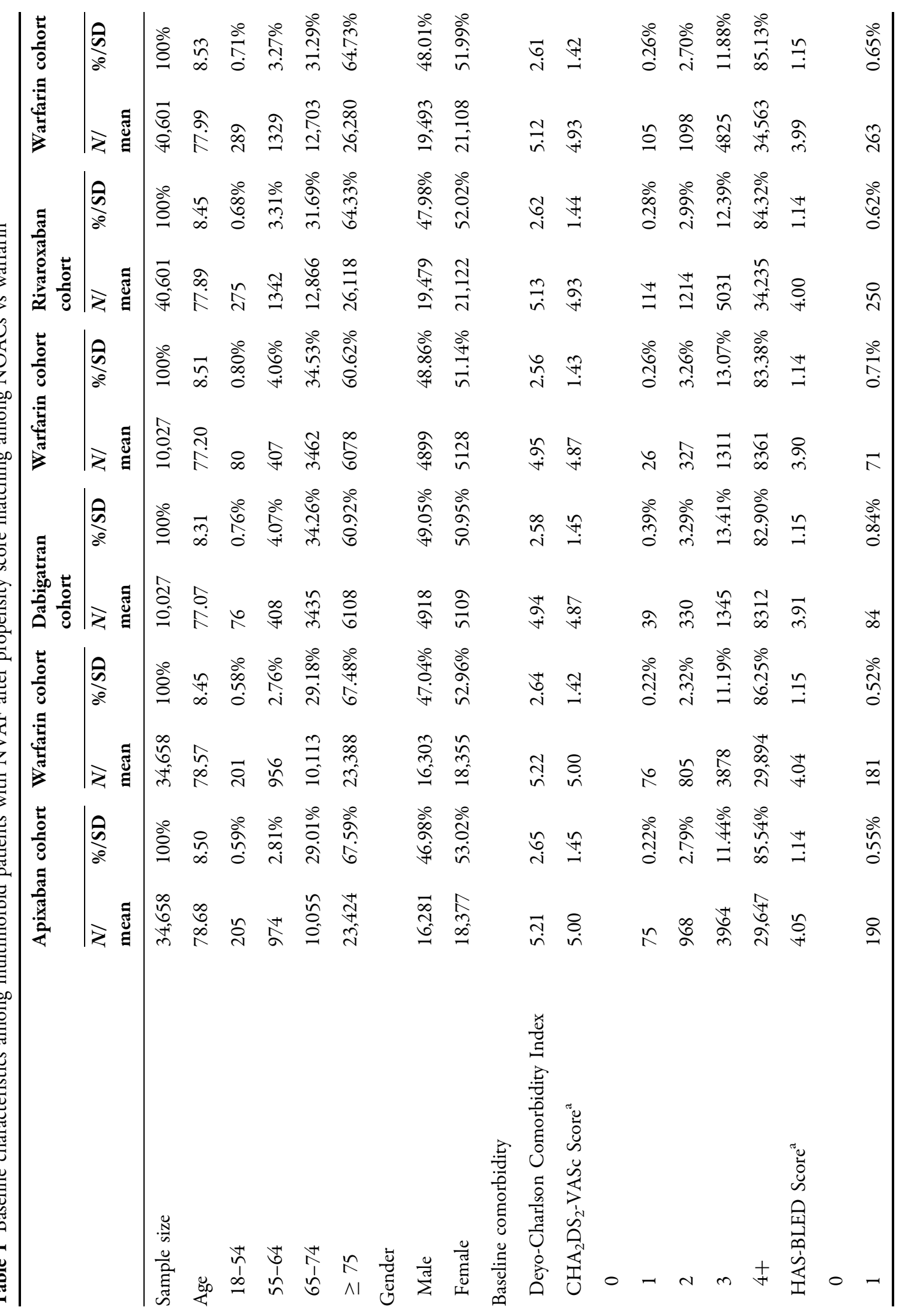




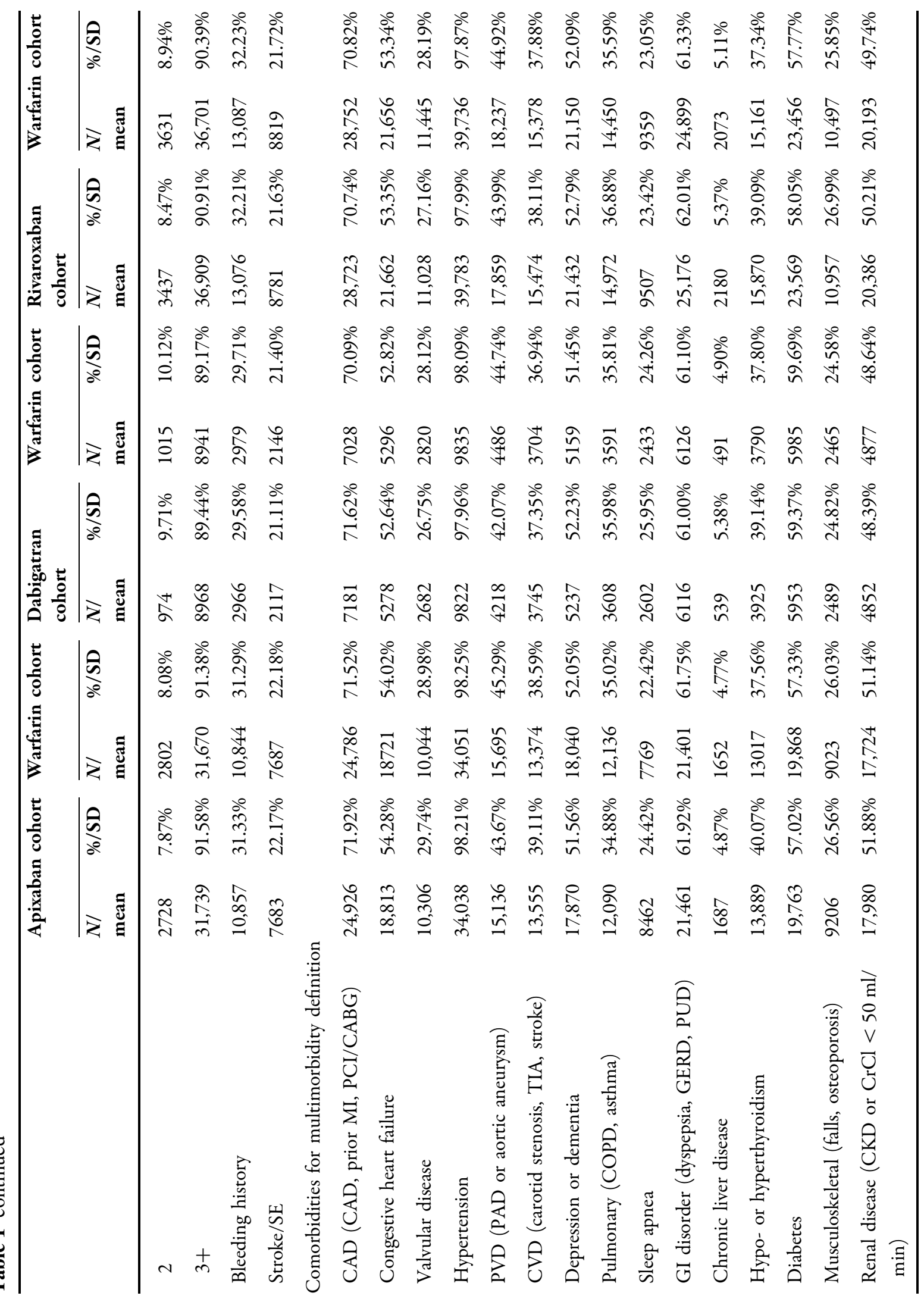




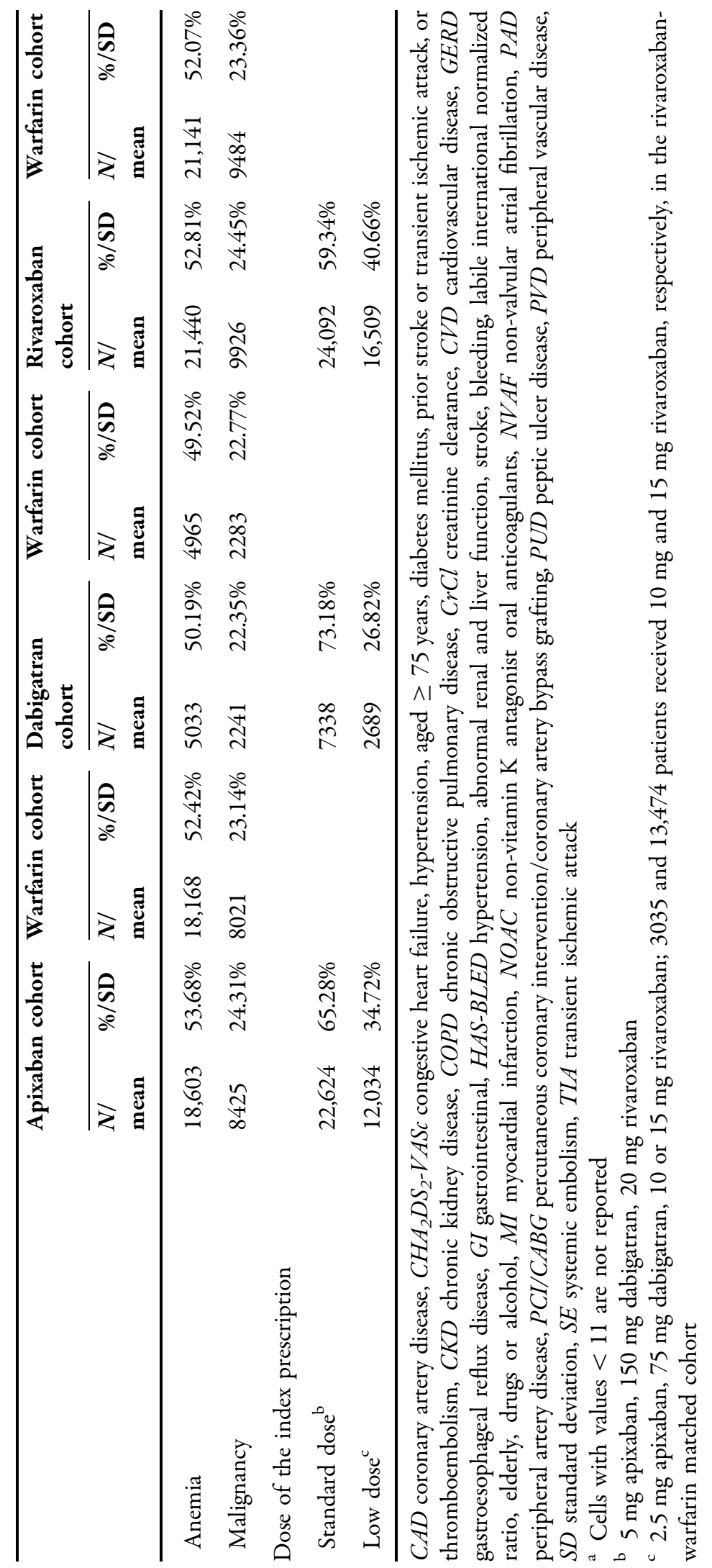




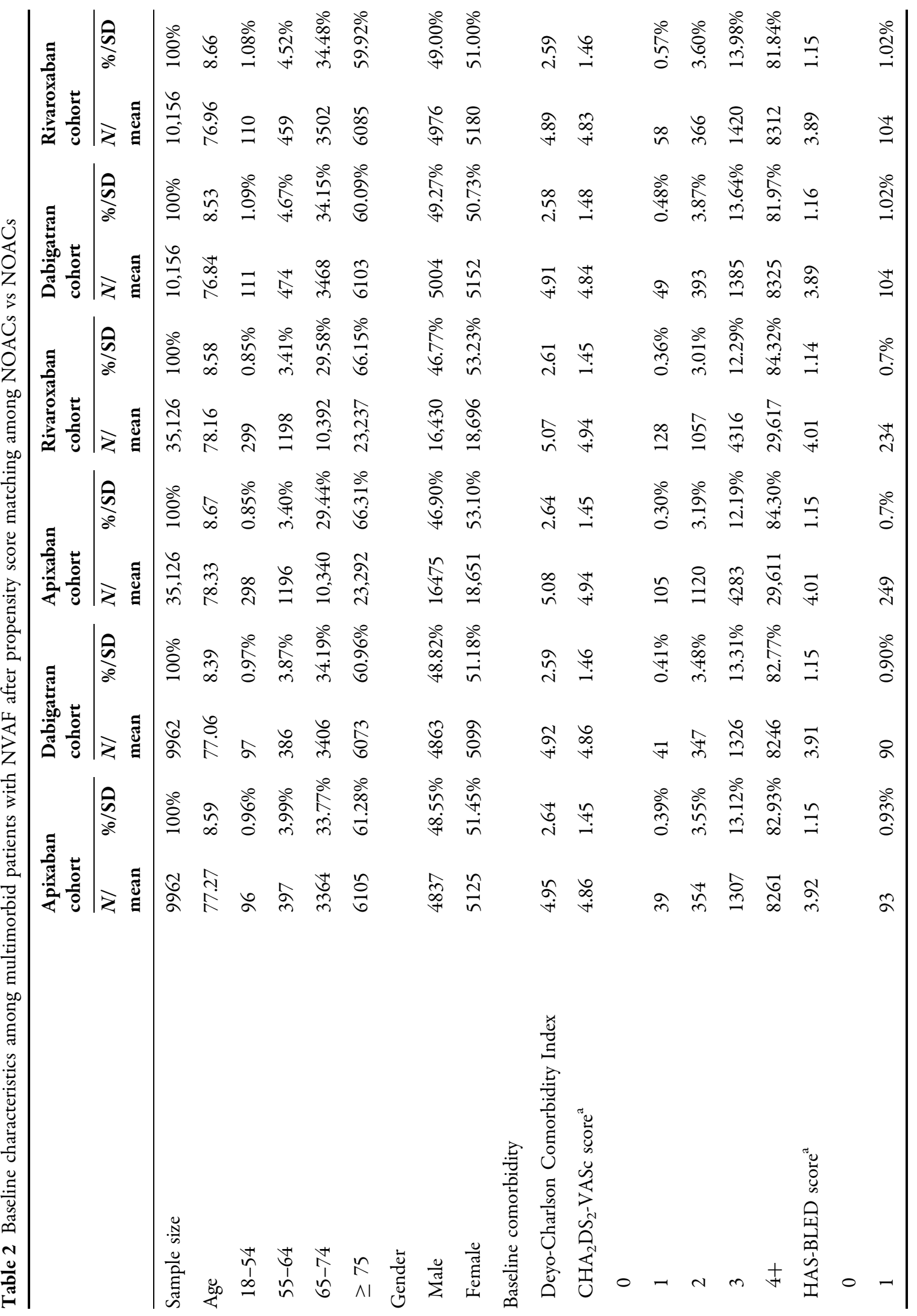




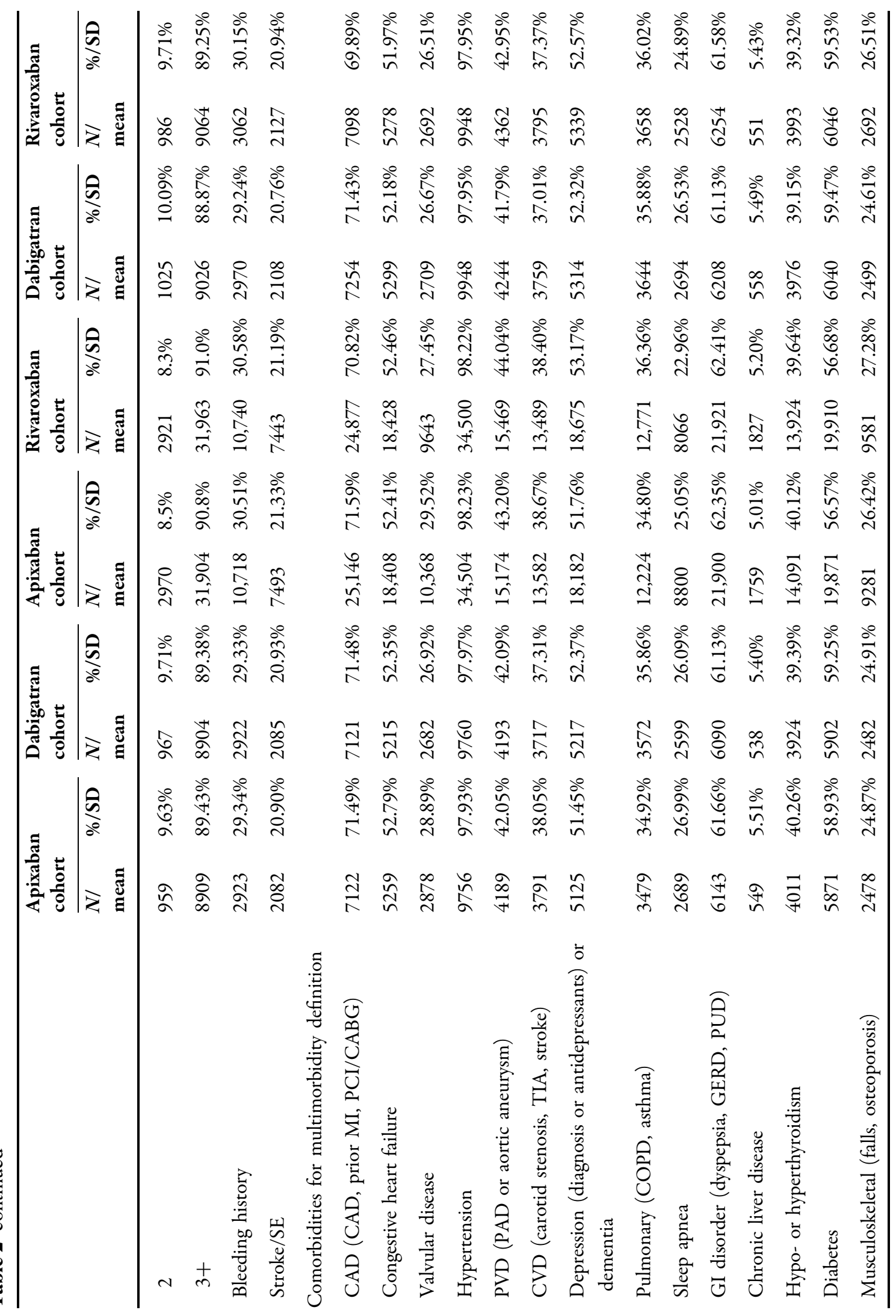




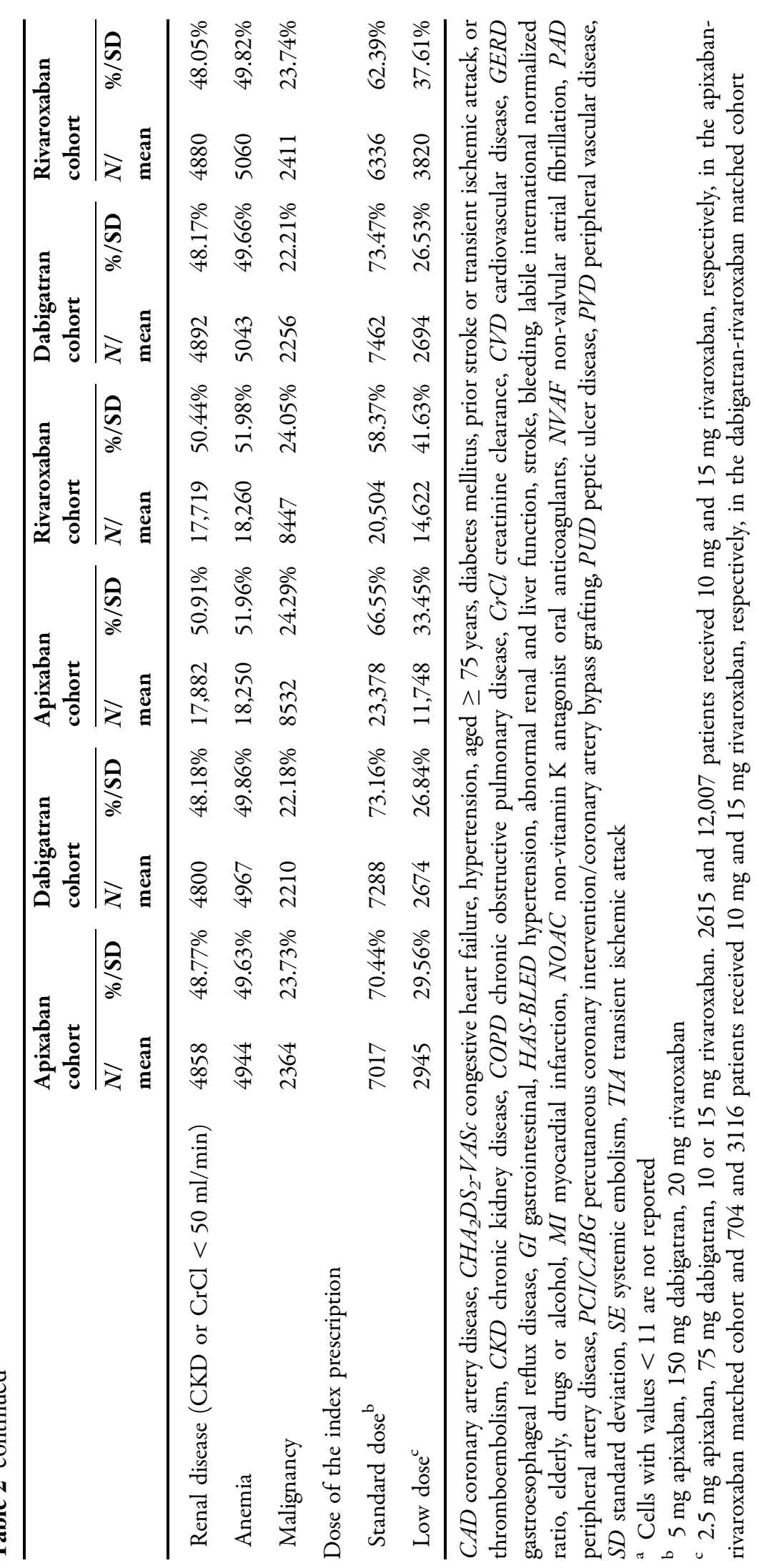




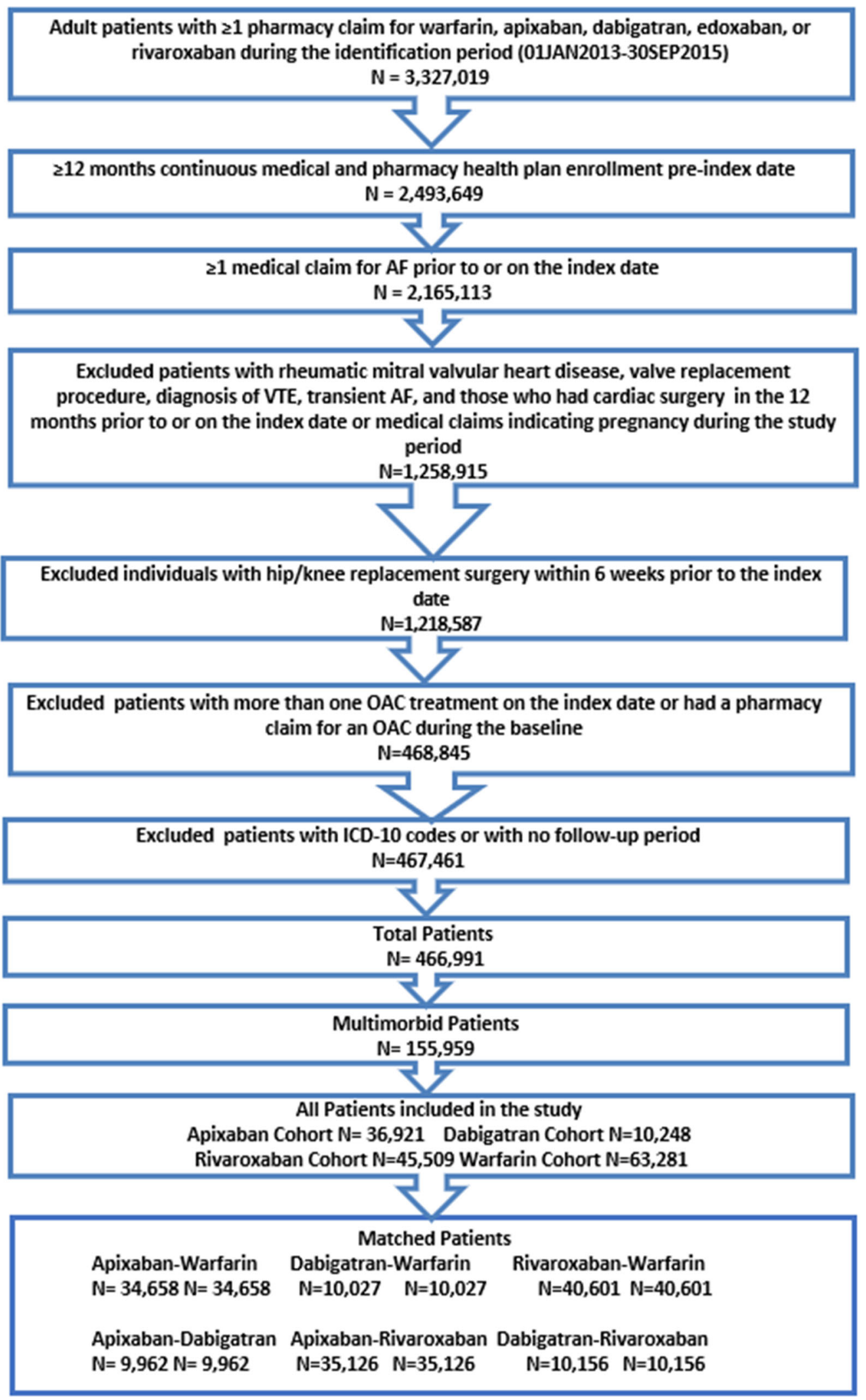


4Fig. 1 Study selection. AF atrial fibrillation; Clinical Modification, OAC oral anticoagulant, VTE venous thromboembolism

1 day post-index date to the first of 30 days after discontinuation, switch date, death, end of continuous medical or pharmacy plan enrollment, or end of study.

\section{Statistical Methods}

Propensity score matching (PSM) was conducted between NOAC and warfarin cohorts and between the NOAC cohorts. Patients were matched 1:1 in each dataset based on the propensity scores generated by logistic regression using demographics, Charlson Comorbidity Index (CCI) scores [14], comorbidities that were documented risk factors for stroke/SE or $\mathrm{MB}$, and baseline co-medications. Patients were matched by nearest neighbor matching without replacement using a caliper of 0.01 . Covariate balance was checked through standardized differences, with a threshold of $10 \%$ [15]. Patients from the five matched datasets were pooled for the final analysis.

Risk of stroke/SE and risk of MB were evaluated using Cox proportional hazard models, with robust sandwich estimates $(\alpha=0.05)$ [16]. OAC treatment was included as the independent variable; no other covariates were included as the cohorts were balanced.

All statistical analyses were performed using SAS version 9.4.

\section{Subgroup Analyses}

An interaction analysis was conducted between treatment and comorbidity level among patients with $6-8$ comorbidities vs patients with $9+$ comorbidities. The statistical significance $(p<0.10)$ of the interaction between treatment and comorbidity level was evaluated. Further, PSM and Cox proportional hazard models were separately completed for patients prescribed standard dose NOACs (apixaban $5 \mathrm{mg}$, dabigatran $150 \mathrm{mg}$, rivaroxaban $20 \mathrm{mg}$ ) and low dose
NOACs (apixaban $2.5 \mathrm{mg}$, dabigatran $75 \mathrm{mg}$, rivaroxaban 10 or $15 \mathrm{mg}$ ).

\section{Compliance with Ethics Guidelines}

Since the core study described herein did not involve the collection, use, or transmittal of individual identifiable data, institutional review board (IRB) approval to conduct this study was not required. The security of the data meets the requirements of the Health Insurance Portability and Accountability Act (HIPAA) of 1996. The study protocol was reviewed and determined to be exempt from the Office for Human Research Protections (OHRP's) Regulations for the Protection of Human Subjects (45 CFR 46) under the following category: Exemption 4: Research involving the collection or study of existing data, documents, records, pathological specimens, or diagnostic specimens, if these sources are publicly available or if the information is recorded by the investigator in such a manner that subjects cannot be identified, directly or through identifiers linked to the subjects.

\section{RESULTS}

After applying the selection criteria, 33.4\% $(n=155,959)$ of the NVAF population newly initiated on OACs $(n=466,991)$ had multimorbidity; 36,921 (23.7\%) initiated apixaban, $10,248 \quad(6.6 \%)$ dabigatran, 45,509 (29.2\%) rivaroxaban, and 63,281 (40.6\%) warfarin (Fig. 1). The mean number of comorbidities ranged from 7.5 to 7.8 across the drug cohorts. Before PSM, apixaban patients were older and warfarin patients had higher $\mathrm{CCI}, \mathrm{CHA}_{2} \mathrm{DS}_{2^{-}}$ VASc, and HAS-BLED scores compared to NOAC patients (Supplementary Table S2).

The unadjusted incidence rates of stroke/SE per 100 person-years were 3.7 (warfarin), 2.4 (apixaban), 2.8 (dabigatran), and 2.4 (rivaroxaban). The unadjusted rates for MB per 100 person-years were 11.5 (warfarin), 6.9 (apixaban), 7.2 (dabigatran), and 10.5 (rivaroxaban) (Supplementary Table S3).

After PSM, 34,658 apixaban-warfarin, 10,027 dabigatran-warfarin, and 40,601 rivaroxabanwarfarin patient pairs were studied. Matching 
for NOAC comparisons included 9962 patient pairs for the apixaban-dabigatran, 35,126 pairs for the apixaban-rivaroxaban, and 10,156 pairs for the dabigatran-rivaroxaban cohorts (Fig. 1). Coronary artery disease, hypertension, and GI disorders were among some of the most prevalent comorbid conditions. The baseline characteristics of the matched populations are shown in Tables 1 and 2.

\section{NOAC vs Warfarin Comparisons}

Compared to warfarin, apixaban and rivaroxaban were associated with a lower risk of stroke/ SE (apixaban: hazard ratio [HR] 0.63, 95\% confidence interval [CI] 0.54-0.74; rivaroxaban: HR 0.70, 95\% CI 0.64-0.77). Dabigatran was associated with a similar risk of stroke/SE (HR 0.91, 95\% CI 0.75-1.10) compared to warfarin. Apixaban and dabigatran were associated with a lower risk of $\mathrm{MB}$ compared to warfarin (HR 0.61, 95\% CI 0.56-0.67; HR 0.75, 95\% CI 0.66-0.86, respectively) while rivaroxaban patients had a higher risk of MB (HR 1.06, 95\% CI 1.01-1.12; Fig. 2a).

\section{NOAC vs NOAC Comparisons}

Compared to dabigatran, apixaban was associated with a lower risk of stroke/SE (HR 0.81, 95\% CI 0.65-1.00) and MB (HR 0.82, 95\% CI 0.69-0.98). Dabigatran was associated with a higher risk of stroke/SE versus rivaroxaban (HR $1.29,95 \%$ CI 1.02-1.62). Apixaban and dabigatran were associated with lower risks of $\mathrm{MB}$ versus rivaroxaban (HR 0.57, 95\% CI 0.52-0.63; HR 0.72 , 95\% CI $0.64-0.81$, respectively) (Fig. 2b). The Kaplan-Meier curves for cumulative incidence rates of stroke/SE and MB in the matched populations are shown in Supplementary Figs. S1A-S1L.

\section{Subgroup Analyses}

Significant interactions were observed for treatment and number of comorbidities among some of the analyses for stroke/SE and MB. For apixaban vs warfarin and apixaban vs rivaroxaban, patients with 6-8 comorbidities experienced a
Fig. 2 Incidence rates and hazard ratios for stroke and major bleeding: a NOACs vs warfarin; b NOACs vs NOACs. CI confidence interval, GI gastrointestinal, NOAC non-vitamin $\mathrm{K}$ antagonist oral anticoagulant, SE systemic embolism. ${ }^{a}$ The upper confidence interval was rounded from 0.999 to 1

greater relative magnitude of reduction in the risk of $\mathrm{MB}$ compared to patients with 9+ comorbidities (Tables 3 and 4). A significant interaction was also found for apixaban vs rivaroxaban for stroke/SE $(p=0.064)$ where apixaban patients with 6-8 comorbidities had a similar risk of stroke/SE, but apixaban patients with 9+ comorbidities trended towards a lower risk of stroke/SE compared with rivaroxaban patients (Table 4). The results of the dose analyses were generally consistent with the main analysis. In the standard dose comparison, all NOACs demonstrated a lower risk of stroke/SE compared to warfarin (Supplementary Figs. S2 and S3).

\section{DISCUSSION}

This ARISTOPHANES subgroup analysis is the largest study to date that evaluates the effectiveness and safety of OAC treatment in a multimorbid (at least six comorbidities) patient population with NVAF. Overall, NOACs were found to be associated with varying risk of stroke/SE and $\mathrm{MB}$ compared to warfarin and differential risk of stroke/SE and $\mathrm{MB}$ was also observed between NOACs. Further analyses in key subgroups, including NOAC low- and standard-dose populations and patients with $6-8$ vs $9+$ comorbidities, showed generally consistent findings.

Multimorbidity is common in patients with NVAF, and proportionally becoming more prevalent in recent years [17]. The presence of multimorbidity is associated with an increased risk of stroke, bleeding, and death [17]. The results of the present study are similar to the findings from related post hoc analyses of the ARISTOTLE trial. Therein, patients were included on the basis of the number of comorbidities and classified as no multimorbidity (0-2 
a

Comparator Warfarin

Incidence per 100 person-years Hazard Ratio $(95 \% \mathrm{Cl})$ ( $N=34,658$ vs. $N=34,658$ )

Apixaban vs Warfarin $(\mathrm{N}=\mathbf{3 4 , 6 5 8}$ vs. $\mathrm{N}=\mathbf{3 4 , 6 5 8 )}$
$2.40 \quad 3.47$

\begin{tabular}{|c|c|}
\hline Stroke/SE & 2.40 \\
\hline Ischemic & 1. \\
\hline Hemorrhagic & 0. \\
\hline Systemic Embolism & 0.10 \\
\hline Major Bleeding & 7. \\
\hline Gl Bleeding & 3.43 \\
\hline Intracranial Hemorrhage & 0. \\
\hline Other sites & 3. \\
\hline Dabigatran vs Warfarin & \\
\hline
\end{tabular}

Dabigatran vs Warfarin

Stroke/SE

\begin{tabular}{|c|}
\hline Stroke/SE \\
\hline Ischemic \\
\hline Hemorrhagic \\
\hline Systemic Embolism \\
\hline Major Bleeding \\
Gl Bleeding \\
\hline Intracranial Hemorrhage \\
\hline Other sites \\
\hline
\end{tabular}

Rivaroxaban vs Warfarin

Stroke/SE

Ischemic

Hemorrhagic

Systemic Embolism

Major Bleeding

GI Bleeding

Intracranial Hemorrhage

Other sites

$1.95 \quad 2.50$

$0.37 \quad 0.81$

$\begin{array}{ll}0.10 & 0.17 \\ 7.05 & 10.54\end{array}$

$\begin{array}{ll}0.06 & 10.54 \\ 3.43 & 4.95\end{array}$

$0.82 \quad 1.42$

$3.12 \quad 4.77$

$(\mathrm{N}=10,027$ vs. $\mathrm{N}=10,027)$

$\begin{array}{lll}2.79 & 3.07 & 0.91(0.75-1.10)\end{array}$

$\begin{array}{ll}2.34 & 2.27 \\ 0.33 & 0.73\end{array}$

$0.33 \quad 0.73$

$\begin{array}{ll}0.12 & 0.08 \\ 7.27 & 9.67\end{array}$

$4.40 \quad 4.45$
0.74

9.67
4.45

$\begin{array}{ll}0.74 & 1.31 \\ 2.59 & 4.49\end{array}$

( $N=40,601$ vs. $N=40,601$ )

$2.33 \quad 3.27$

$1.79 \quad 2.34$

$0.40 \quad 0.80$

$0.14 \quad 0.18$

$10.94 \quad 10.17$

6.08

4.71

0.76

1.41

$4.95 \quad 4.63$

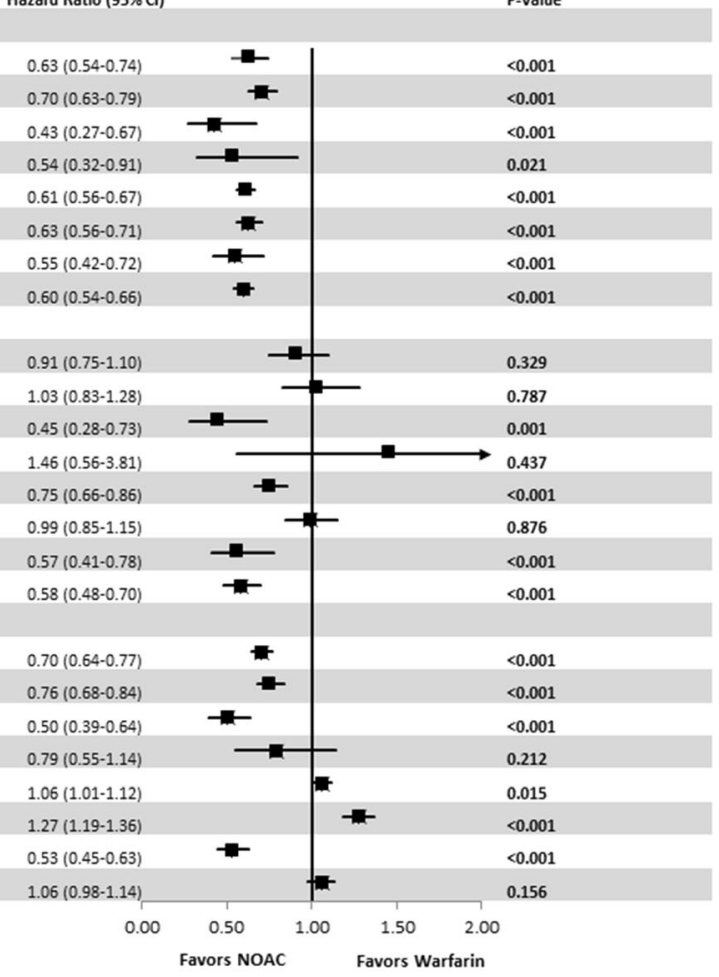

b

Comparator Reference

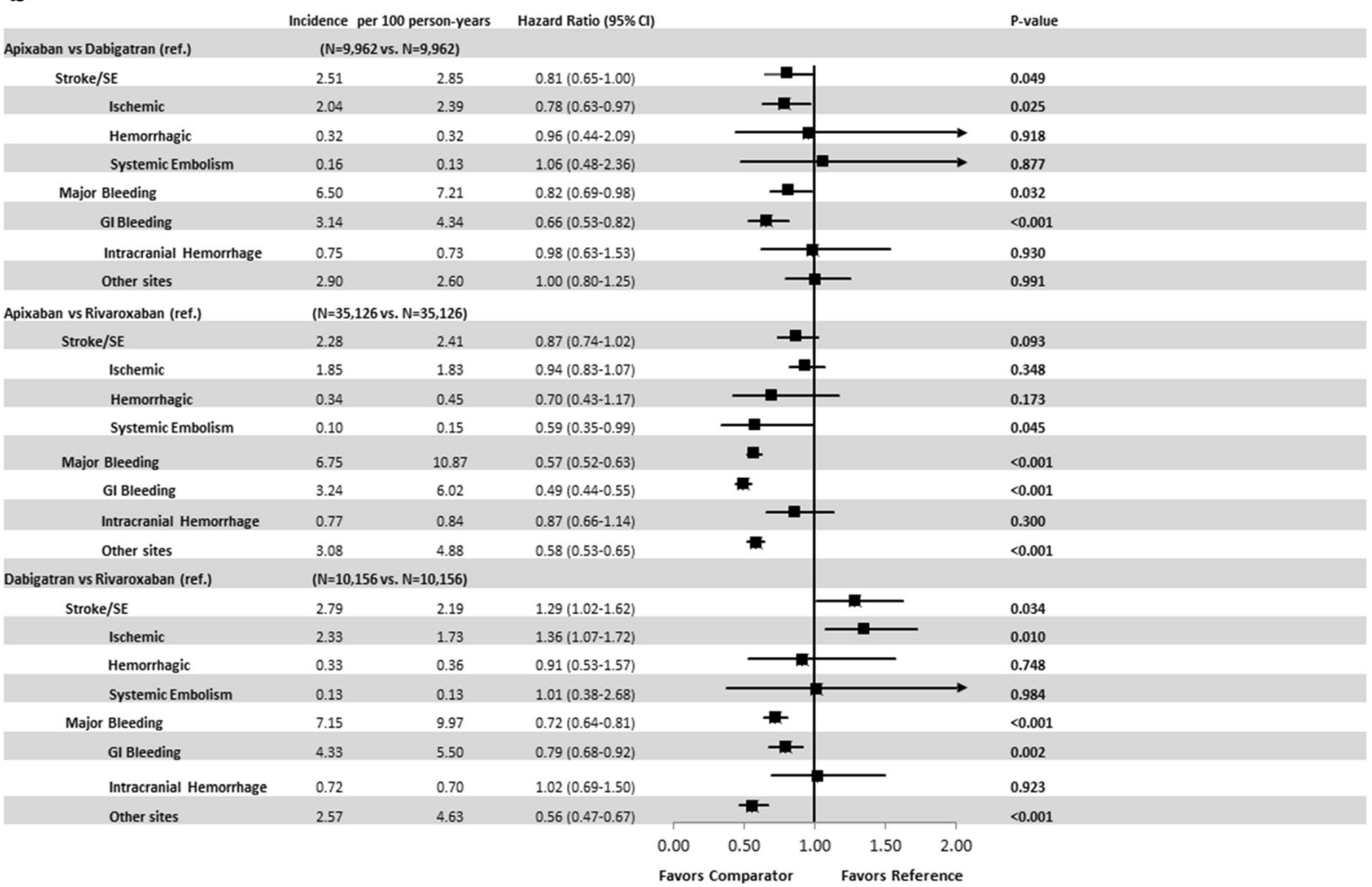




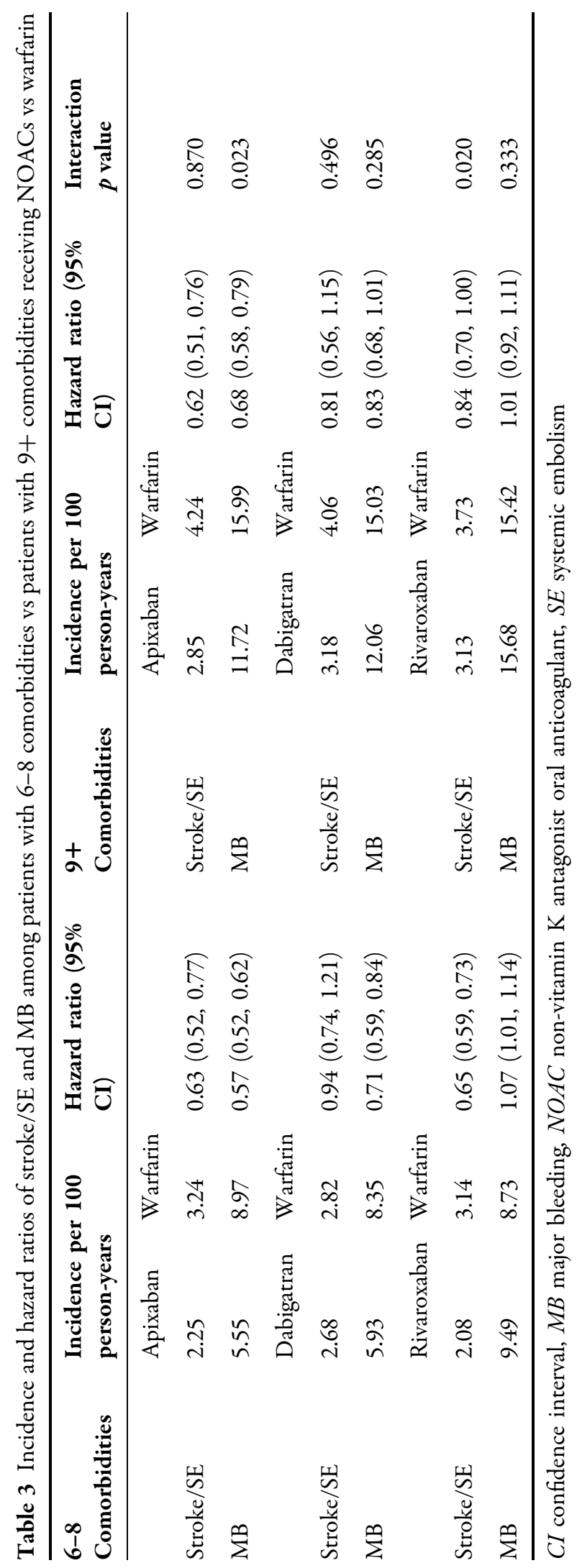




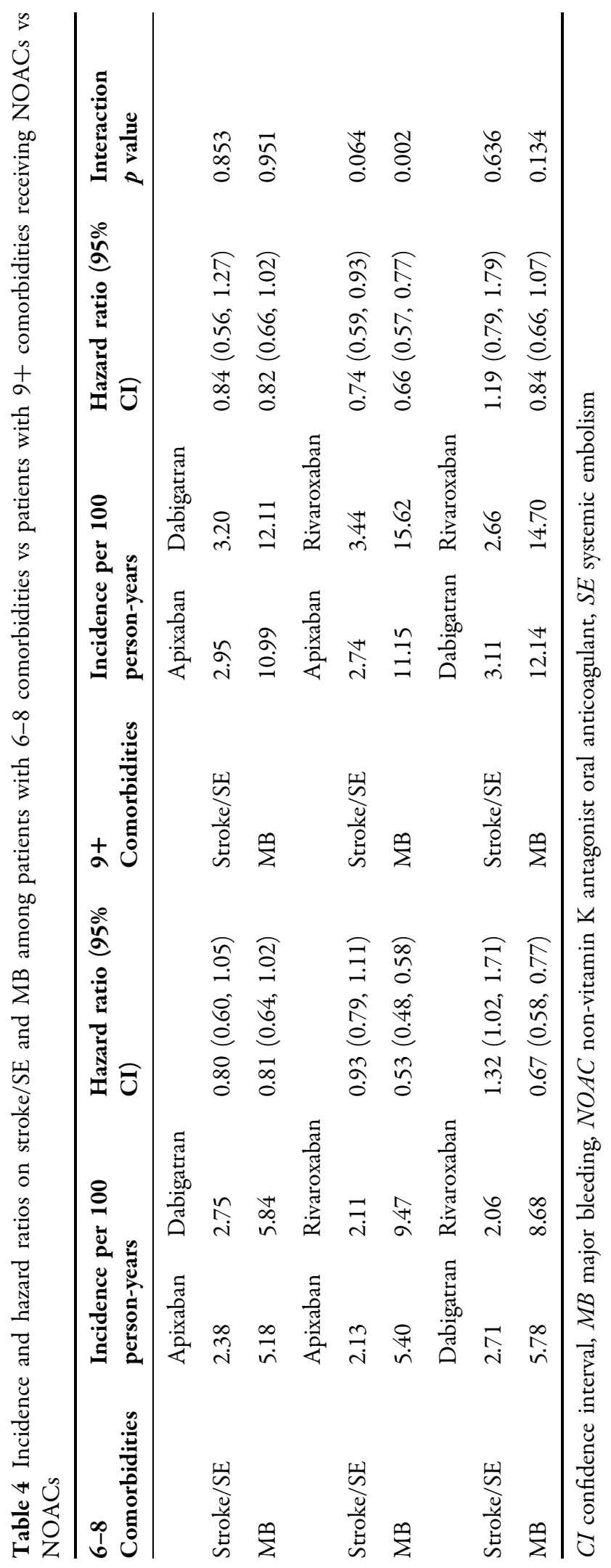


comorbidities), moderate multimorbidity (3-5 comorbidities), and high multimorbidity (at least 6 comorbidities), and the risks of stroke/SE and $\mathrm{MB}$ were elevated for patients in the high and moderate multimorbidity groups compared with the no multimorbidity group [9]. The ARISTOTLE post hoc analysis also found that multimorbid apixaban patients trended toward a lower risk of stroke/SE and $\mathrm{MB}$ compared to warfarin, which is consistent with both the main trial results and the present study [9].

Few real-world retrospective studies have evaluated the impact of OAC treatment in patients with $\mathrm{AF}$ and multimorbidity. One study done by Mentias et al. assessed the effectiveness of dabigatran, rivaroxaban, and warfarin among Medicare patients with AF where multimorbidity was indicated by $\mathrm{CHA}_{2} \mathrm{DS}_{2-}$ VASc, HAS-BLED, and Gagne comorbidity scores rather than number of comorbid conditions at baseline [18]. Across the three comorbidity scores, the authors found that standarddose dabigatran patients did not have a significantly different risk of stroke or MB compared to warfarin. This is inconsistent with the results of both the standard-dose subgroup analysis of this study and results from the RE-LY trial, which showed that dabigatran had a significantly lower risk of stroke/SE and MB compared to warfarin [19]. Mentias et al. (2018) found that standard-dose rivaroxaban patients had a higher risk of $\mathrm{MB}$ compared to dabigatran patients across all three scores, which is consistent with results of our main analysis and standard-dose subgroup analysis [18]. The discrepancy in trends may be due to the difference in defining multimorbidity.

Compared to previous studies, this ARISTOPHANES subgroup analysis provides a larger sample of patients with NVAF and multimorbidity from a more representative population, including both Medicare and commercially insured patients. The results are consistent with available RCT literature in showing that NOACs are associated with favorable benefit/risk profile when compared to warfarin.

\section{Limitations}

For this retrospective observational study, only statistical associations could be concluded, not causal relationships. Although cohorts were matched through PSM, there were potential residual confounders. As a result of the nature of claims studies, outcome measures could only be based on International Classification of Diseases-Ninth Revision-Clinical Modification (ICD-9-CM) codes without further adjustment with precise clinical criteria. In addition, laboratory values, such as hemoglobin or international normalized ratio (INR) measurements, are not available in the dataset so time in therapeutic range for patients prescribed warfarin was indeterminable. Nonetheless, the inclusion of patients with potentially poorer quality control of warfarin therapy in everyday clinical practice may enable the study findings to better reflect real-world situations. Additionally, previously verified mortality information was only available for Medicare patients, and although $62 \%$ of stroke deaths occur outside of an acute hospital setting, it is unlikely that the proportion of deaths would differ by cohort $[20,21]$.

This study used both the distribution of comorbidities at baseline and previously published ARISTOTLE trial subgroup analyses to define multimorbidity, but definition of multimorbidity differs from some other studies and presents a challenge to compare our findings with those of other studies. While all patients were required to have at least six comorbidities, the severity of conditions remained unknown and may not be equal among patients. Moreover, unobserved heterogeneity may exist across the five data sources. Although some of the datasets contain information from different insurance plans that do not overlap at the plan level, others are employer-based claims datasets which may contain duplicate patient records when pooled together. However, the number of such duplicates is likely to be low-on the basis of a published estimate of $0.5 \%$-and therefore unlikely to have any significant effect on the results [22]. Finally, the results may not reflect the overall multimorbid NVAF population in the USA because the study did not include 
uninsured patients and those solely covered by other public health insurance plans.

\section{CONCLUSIONS}

In the largest observational study of patients with NVAF and multimorbidity, we show that among patients with NVAF and six or more comorbid conditions, NOACs were associated with varying risk of stroke/SE and $\mathrm{MB}$ compared to warfarin and to each other. Rather than a "one drug fits all" approach, our results may be useful for selecting appropriate OAC treatment for multimorbid patients.

\section{ACKNOWLEDGEMENTS}

Funding. Sponsorship for this study and the journal's rapid service fee was provided by Bristol-Myers Squibb Company and Pfizer Inc.

Authorship. All named authors meet the International Committee of Medical Journal Editors (ICMJE) criteria for authorship for this article, take responsibility for the integrity of the work as a whole, and have given their approval for this version to be published.

Medical Writing, Editorial, and Other Assistance. Editorial assistance in the preparation of this article was provided by Michael Moriarty of STATinMED Research. Support for this assistance was funded by Bristol-Myers Squibb Company and Pfizer Inc.

Disclosures. Steven Deitelzweig is a consultant for BMS/Pfizer, Daiichi-Sankyo, Portola, and Boehringer Ingelheim, and has been on the speakers' bureau for Janssen, Bristol Myers Squibb Company/Pfizer Inc., and Boehringer Ingelheim. Gregory Y.H. Lip is a consultant for Bayer/Janssen, BMS/Pfizer, Medtronic, Boehringer Ingelheim, Novartis, Verseon and DaiichiSankyo. Speaker for Bayer, BMS/Pfizer, Medtronic, Boehringer Ingelheim, and Daiichi-Sankyo. No fees are directly received personally. Huseyin Yuce has nothing to disclose. Allison
Keshishian is a paid employee of STATinMED Research which is a paid consultant to BristolMyers Squibb Company and Pfizer Inc. Amiee Kang, Amol Dhamane, Christian Klem, Lisa Rosenblatt and Jenny Jiang are paid employees of Bristol-Myers Squibb Company. Xuemei Luo and Jack Mardekian are paid employees of Pfizer Inc.

Compliance with Ethics Guidelines. Since the core study described herein did not involve the collection, use, or transmittal of individual identifiable data, institutional review board (IRB) approval to conduct this study was not required. The security of the data meets the requirements of the Health Insurance Portability and Accountability Act (HIPAA) of 1996. The study protocol was reviewed and determined to be exempt from the Office for Human Research Protections (OHRP's) Regulations for the Protection of Human Subjects (45 CFR 46) under the following category: Exemption 4: Research involving the collection or study of existing data, documents, records, pathological specimens, or diagnostic specimens, if these sources are publicly available or if the information is recorded by the investigator in such a manner that subjects cannot be identified, directly or through identifiers linked to the subjects. This study was performed in accordance with the Helsinki Declaration of 1964, and its later amendments.

Data Availability. The raw data on which these analyses were based were obtained from the Centers for Medicare \& Medicaid Services (CMS) and four US commercial claims databases: the IBM MarketScan ${ }^{\circledR}$ Commercial Claims and Encounter Database, the IQVIA PharMetrics Plus ${ }^{\mathrm{TM}}$ Database, the Optum Clinformatics $^{\mathrm{TM}}$ Data Mart, and the Humana Research Database. These data are available from the respective organizations upon request.

Open Access. This article is licensed under a Creative Commons Attribution-NonCommercial 4.0 International License, which permits any non-commercial use, sharing, adaptation, distribution and reproduction in any medium or format, as long as you give appropriate credit 
to the original author(s) and the source, provide a link to the Creative Commons licence, and indicate if changes were made. The images or other third party material in this article are included in the article's Creative Commons licence, unless indicated otherwise in a credit line to the material. If material is not included in the article's Creative Commons licence and your intended use is not permitted by statutory regulation or exceeds the permitted use, you will need to obtain permission directly from the copyright holder. To view a copy of this licence, visit http://creativecommons.org/licenses/by$\mathrm{nc} / 4.0 /$.

\section{REFERENCES}

1. Zoni-Berisso M, Lercari F, Carazza T, Domenicucci S. Epidemiology of atrial fibrillation: European perspective. Clin Epidemiol. 2014;6:213.

2. De Caterina R, Husted S, Wallentin L, et al. Vitamin $\mathrm{K}$ antagonists in heart disease: current status and perspectives (Section III). Position paper of the ESC Working Group on Thrombosis-Task Force on Anticoagulants in Heart Disease. Thromb Haemost. 2013;110:1087-107.

3. Ruff CT, Giugliano RP, Braunwald E, et al. Comparison of the efficacy and safety of new oral anticoagulants with warfarin in patients with atrial fibrillation: a meta-analysis of randomised trials. Lancet. 2014;383:955-62.

4. January CT, Wann LS, Calkins H, et al. 2019 AHA/ ACC/HRS focused update of the 2014 AHA/ACC/ HRS guideline for the management of patients with atrial fibrillation: a report of the American College of Cardiology/American Heart Association Task Force on Clinical Practice Guidelines and the Heart Rhythm Society. J Am Coll Cardiol. 2019;74(1): 104-32.

5. Salive ME. Multimorbidity in older adults. Epidemiol Rev. 2016;35:75-83.

6. Parks AL, Fang MC. Anticoagulation in older adults with multimorbidity. Clin Geriatr Med. 2016;32: 331-46.

7. Vanbeselaere V, Truyers C, Elli S, et al. Association between atrial fibrillation, anticoagulation, risk of cerebrovascular events and multimorbidity in general practice: a registry-based study. BMC Cardiovasc Dis. 2016;16(1):61.
8. Jani BD, Nicholl BI, McQueenie R, et al. Multimorbidity and co-morbidity in atrial fibrillation and effects on survival: findings from UK Biobank cohort. EP Europace. 2017;20(FI_3):f329-36.

9. Alexander KP, Brouwer MA, Mulder H, et al. Outcomes of apixaban versus warfarin in patients with atrial fibrillation and multi-morbidity: insights from the ARISTOTLE trial. Am Heart J. 2019;208: 123-31.

10. Li X, Deitelzweig S, Keshishian A, et al. Effectiveness and safety of apixaban versus warfarin in nonvalvular atrial fibrillation patients in "real-world" clinical practice. A propensity-matched analysis of 76,940 patients. Thromb Haemost. 2017;117(6): 1072-82.

11. Lip GYH, Keshishian A, Li X, et al. Effectiveness and safety of oral anticoagulants among non-valvular atrial fibrillation patients: the ARISTOPHANES study. Stroke. 2018;49(12):2933-44.

12. Thigpen JL, Dillon C, Forster KB, et al. Validity of international classification of disease codes to identify ischemic stroke and intracranial hemorrhage among individuals with associated diagnosis of atrial fibrillation. Circ Cardiovasc Qual Outcomes. 2015;8(1):8-14.

13. Cunningham A, Stein CM, Chung CP, Daugherty JR, Smalley WE, Ray W. An automated database case definition for serious bleeding related to oral anticoagulant use. Pharmacoepidemiol Drug Saf. 2011;20(6):560-6.

14. Charlson ME, Pompei P, Ales KL, MacKenzie CR. A new method of classifying prognostic comorbidity in longitudinal studies: development and validation. J Chronic Dis. 1987;40(5):373-83.

15. Austin PC. Balance diagnostics for comparing the distribution of baseline covariates between treatment groups in propensity-score matched samples. Stat Med. 2009;28(25):3083-3107.

16. Austin PC. The use of propensity score methods with survival or time-to-event outcomes: reporting measures of effect similar to those used in randomized experiments. Stat Med. 2014;33(7): 1242-58.

17. Proietti M, Marzona I, Vannini T, et al. Long-term relationship between atrial fibrillation, multimorbidity and oral anticoagulant drug use. Mayo Clin Proc. 2019;94(12):2427-36.

18. Mentias A, Shantha G, Chaudhury P, Sarrazin MSV. Assessment of outcomes of treatment with oral anticoagulants in patients with atrial fibrillation and multiple chronic conditions: a comparative 
effectiveness analysis. JAMA Netw Open. 2018;1(5): e182870-e182870.

19. Connolly SJ, Ezekowitz MD, Yusuf S, et al. Dabigatran versus warfarin in patients with atrial fibrillation. New Engl J Med. 2009;361(12):1139-51.

20. Jarosek S. Death Information in the Research Identifiable Medicare Data. ResDAC. https://www. resdac.org/articles/death-information-researchidentifiable-medicare-data. Accessed 1 Mar 2021
21. Virani SS, Alonso A, Aparicio HJ, et al. Heart disease and stroke statistics-2021 update: a report from the American Heart Association. Circulation. 2021;143(8):e254-743. https://doi.org/10.1161/ CIR.0000000000000950.

22. Broder MS, Neary MP, Chang E, Cherepanov D, Katznelson L. Treatments, complications, and healthcare utilization associated with acromegaly: a study in two large United States databases. Pituitary. 2014;17(4):333-41. 